\title{
Carrefour de l'apprentissage des langues : le persan, l'anglais et le français
}

Rozita ILANI, Université Azad d'Arak, Iran

\section{Introduction}

On s'accorde généralement sur le fait que les structures syntaxiques de la langue maternelle des apprenants du français ont un impact non négligeable sur leurs performances en FLE (Roux ; Krashen et Terrell 42 ; Carrasco-Ortiz et Frenck-Mestre 36 ; Dubois et al.). Cet article élargit cette thèse et traite des influences de la langue maternelle ainsi que celles de l'apprentissage d'une langue étrangère déjà acquise sur le FLE (Farkamekh).

L'Iran est un pays où à côté du persan, langue officielle, on est attiré à apprendre une deuxième et même une troisième langue étrangère pour des raisons diverses. Actuellement, la deuxième langue étrangère dans ce pays est la langue anglaise. A la suite de l'internationalisation de l'anglais après la seconde guerre mondiale, cette langue est devenue, en Iran, la première langue étrangère et s'est substituée au français. Mais, le français exerce toujours une attraction chez les apprenants de langues étrangères. Dans ce milieu trilingue, l'interférence des langues fait apparaitre des erreurs linguistiques dont nous traiterons certaines dans ce travail. Notre intérêt pour ce travail remonte à nos expériences d'enseignement de FLE qui nous ont encouragés à étudier les influences du persan ainsi que de l'anglais sur l'apprentissage du français chez des apprenants persanophones.

La plupart des enseignants de FLE ont constaté que depuis l'introduction de la notion de compétence de communication dans l'enseignement du français langue étrangère, la grammaire semble reléguée au second plan. Cependant, les méthodes d'enseignements de FLE présentent toujours des activités grammaticales. Ce paradoxe nous a conduits à nous interroger sur l'analyse des facteurs grammaticaux qui influencent le procès d'apprentissage de FLE en Iran. Pour limiter notre champ de recherche, cet article se focalise sur l'apprentissage des verbes français et des erreurs linguistiques des apprenants dans ce domaine. Il se concentre sur les problèmes principaux d'apprentissage de verbe chez des étudiants débutants iraniens au niveau de licence du français langue étrangère. Nous considérons deux possibilités pour l'apparition des erreurs : l'influence du persan (L1) d'une part et d'autre part l'interférence manifestée entre l'anglais (L2) et l'apprentissage récent du français (L3). L'objectif de notre travail est d'identifier et analyser les erreurs produites en langue française par les apprenants persanophones ayant appris l'anglais comme première langue étrangère. Il nous semble pertinent de présenter brièvement certains aspects des 
contacts de langues et leur influence grammaticale. Ce travail permettra de mettre en évidence, du point de vue de l'apprentissage, les mécanismes en jeu dans la construction du savoir langagier de FLE des apprenants. Dans la perspective de l'enseignement, on pourra tenter d'établir une typologie des erreurs linguistiques qui aidera à profiter des aspects positifs et éviter des interférences négatives.

\section{Cadre théorique}

\subsection{Définition du sujet}

Langue maternelle (LM/L1) de la plupart des Iraniens, le persan (farsi) est la langue officielle d'Iran où on peut constater le phénomène de bilinguisme chez ses habitants. Les langues régionales y sont nombreuses et une moitié de la population iranienne parle leur langue maternelle à côté de la langue officielle. Ainsi, des apprenants des langues connaissent souvent les problèmes de multilinguisme. Depuis longtemps et jusqu'à la seconde guerre mondiale, la langue française, considérée comme le symbole intellectuel, était la première langue étrangère en Iran. Après la deuxième guerre mondiale, remplaçant le français (L3), la langue anglaise (L2), comme langue internationale, attire les apprenants et devient la première langue étrangère. Dans le monde actuel, le discours sur la globalisation et l'influence de l'anglais en tant que langue de communication internationale présentent une recrudescence de l'intérêt pour les situations dans lesquelles plusieurs langues sont utilisées. Le changement du statut de français n'empêche pas les apprenants iraniens d'apprendre cette langue comme la deuxième langue étrangère. De nos jours, les apprenants persanophones veulent apprendre le français dans une courte durée en raison de diverses motivations.

Dans ce contexte social, des contacts linguistiques entre le persan, l'anglais et le français causent des interférences et des influences linguistiques de l'une sur l'autre. Le résultat de ces interférences est considérable et mérite des recherches linguistiques, selon chaque langue vivante. On peut profiter des aspects positifs de ces contacts linguistiques dans l'enseignement et l'apprentissage des langues étrangères, surtout le français, objectif principal de cette recherche.

\subsection{Problématique}

Dans les nouvelles méthodologies d'enseignement des langues étrangères de cette décennie, on relève la place de la langue maternelle et/ou des langues étrangères déjà acquises. Là encore, on accorde tout d'abord une belle part à la langue maternelle (Farkamekh ; Roux). Le recours à la langue maternelle ou à d'autres langues connues des apprenants est aujourd'hui non seulement toléré mais souvent encouragé, à condition qu'il 
soit encadré, choisi, raisonné et corresponde à des options pédagogiques et non pas à une quelconque solution de facilité. On note ainsi l'apparition de la notion de médiation linguistique dans le CECR, qui consiste à traiter dans une langue un support proposé dans une autre langue. Les recherches en L2 présentent un consensus sur le fait que les représentations grammaticales en L1 ont une influence sur l'apprentissage morphosyntaxique en L2 (Roux). En effet, l'emploi des règles de la L1 dans la L2 peut faire avancer l'individu dans son acquisition quand la règle de la L1 est similaire à celle de la L2. En utilisant les règles de la L1, une production prématurée de la L2 aide l'individu à participer à une conversation. En contact avec des entrées linguistiques compréhensibles, l'individu peut acquérir plus de compétences de la langue secondaire (Krashen et Terrell 42). Ces nouvelles données bouleversent l'idée dominante qu'il faut rejeter complètement la langue maternelle dans l'enseignement d'une langue seconde. Selon ces recherches récentes, l'utilisation de la langue maternelle n'est pas prohibée dans le procès d'apprentissage de langue étrangère, mais sa fonction cause certainement des contacts avec la langue cible.

A côté des interférences linguistiques entre la langue maternelle et la langue étrangère (souvent l'anglais), il y a des circonstances dans lesquelles on apprend une troisième ou une quatrième langue étrangère. La langue anglaise est devenue la première langue étrangère apprise dans de nombreux pays, comme en Iran. Dans ces conditions, l'apprentissage de la langue française comme seconde langue étrangère s'effectue après celui de l'anglais. C'est alors que des interférences et des contacts de ces trois langues (persan, anglais, français) sont inévitables en milieu d'apprentissage de FLE en Iran.

Afin de décrire l'origine des interférences de ces langues dans notre recherche, il faut tenir compte que l'apprenant iranien estime généralement que l'anglais et le français semblent identiques parce que ces langues utilisent l'alphabet latin. En effet, l'anglais semble plus proche du français que le persan qui s'éloigne de ces deux langues avec l'utilisation de l'alphabet arabe. Les locuteurs persanophones n'ont pas d'antériorité langagière sur certaines parties du discours du français (L3) par rapport à leur langue maternelle (L1). Puisque l'anglais possède des notions similaires, ces apprenants ayant appris l'anglais (L2) auparavant sont susceptibles de mettre en place leurs nouvelles connaissances dans leurs productions langagières du français. En apprenant l'anglais, ils les ajoutent donc à leurs connaissances linguistiques pour faciliter l'apprentissage du français. Si les connaissances préalables sont identiques à celles du français, on économise du temps dans l'apprentissage et on recherche la simplicité dans la production langagière ; dans le cas contraire, des apprenants rencontrent des problèmes, car on est piégé par le phénomène des influences interlinguistiques 
(Farkamekh 12-14). D'autre part, les ressemblances structurelles entre le persan et le français ne sont pas négligeables. Pour citer un exemple, on peut se référer à la règle grammaticale de l'accord du nombre entre le sujet et le verbe qui est identique en persan et en français. Comme la grammaire du persan possède cette notion, on peut étudier l'aspect positif d'interférence du persan et du français. Mais malgré certaines ressemblances syntaxiques entre le persan et le français, l'apprenant iranien, consciemment ou inconsciemment, se réfère généralement à l'anglais et il y a un certain aller-retour entre les savoirs grammaticaux de ces deux langues étrangères. Ces différences et ces ressemblances accentuent les phénomènes de transfert à partir du persan ou de l'anglais.

\subsection{Hypothèses}

Selon les erreurs des apprenants du FLE comme L3 en Iran, cet article veut répondre aux questions : quelle est l'influence de la grammaire persane et l'influence de l'apprentissage préalable de l'anglais sur les erreurs du français chez des apprenants persanophones ? Quelles sont les erreurs grammaticales dues au persan et à l'anglais lors de l'apprentissage des verbes français ? Comment les différences et les ressemblances entre le persan, l'anglais et le français déterminent-elles des erreurs?

Nous faisons l'hypothèse que la source de ces erreurs est l'apprentissage préalable des grammaires persane et anglaise et l'interférence des connaissances grammaticales de ces langues. Une analyse contrastive de L1, L2 et L3 peut révéler l'influence des structures grammaticales du persan et de l'anglais sur l'apprentissage des verbes français. Nous examinons donc cette hypothèse que certaines ressemblances entraînent des transferts positifs alors que d'autres différences posent des problèmes d'interférence.

\subsection{Méthodologie}

On a choisi une cinquantaine d'étudiants en première année de licence d'interprétation de français langue étrangère des universités Azad de Téhéran et d'Arak pendant les années 2012 et 2015. Âgés de dix-huit à trente ans, ces participants faisaient déjà l'étude de l'anglais depuis au moins six ou sept ans dans des cours de deux heures par semaine lors de leur scolarité avant de commencer le français à l'université. Précisons que tous ces participants étaient complètement débutants en français au début de leurs études universitaires.

\subsection{Importance du sujet et des supports historiques}

Le phénomène d'interlangue des langues peut exister dans tous les pays qui possèdent une langue maternelle éloignée de la langue cible par rapport à l'anglais. Cet article peut alors fournir une base de travail, dans sa méthodologie et avec ses limitations, pour envisager d'étudier les influences interlinguistiques dans l'apprentissage de la langue française dans les 
cas où la langue maternelle est éloignée, par sa morphologie et/ou sa typologie des autres langues apprises (anglais, etc.) et à apprendre (français). Le résultat de telles recherches aide des professeurs de FLE à faciliter le procès d'enseigner le français et des apprenants à économiser le temps et atteindre rapidement le but. Les principales recherches portent sur l'interférence des langues et l'influence du bilinguisme sur l'acquisition de L3 (Farkamekh ; Bild et Swain ; Cenoz et al.) et sur l'importance de la L1 dans l'apprentissage de la L2 (Roux ; Krashen et Terrell ; Carrasco-Ortiz et Frenck-Mestre ; Dubois et al.).

\section{Discours}

\subsection{Carrefour des langues}

En Iran, on entreprend l'étude de l'anglais comme première langue étrangère au début de l'enseignement secondaire et cet apprentissage s'adresse à des enfants de onze ans. La plupart des étudiants ont étudié l'anglais comme première langue étrangère avant de débuter l'apprentissage du français à l'université. Cette dominance de l'anglais n'empêche pas les Iraniens d'apprendre le français. Les différents objectifs motivent des apprenants, surtout les adultes, à apprendre cette langue mélodique : objectif culturel comme la littérature française, objectif communicatif, poursuite des études dans des universités françaises et enfin immigration en pays francophones (comme le Canada). L'apprentissage du français consiste donc en une acquisition consciente, méthodique et raisonnée des éléments langagiers. Il est plutôt mécanique chez des adultes persanophones qui ont déjà appris les règles grammaticales de la langue maternelle et de l'anglais et cherchent des règles et des explications grammaticales pour économiser le temps et faciliter l'apprentissage de FLE. Dans les cours des langues étrangères en Iran, on constate couramment l'attachement des élèves adultes à apprendre la grammaire avant tout. L'apprenant iranien considère souvent la grammaire comme le véhicule des compétences orales et écrites de la langue étrangère. Il se sent plus capable quand il exerce ses connaissances grammaticales afin d'améliorer sa compétence orale. Le jalon de ses connaissances grammaticales est posé sur les savoirs grammaticaux préalables de sa langue maternelle et/ou de l'anglais. La base de cette théorie consiste en des recherches linguistiques qui expliquent le rôle de premières langues dans l'acquisition d'une langue seconde. Grâce aux bases linguistiques communes entre les langues, on peut apprendre des savoirs langagiers d'une langue maternelle et les transformer en langue seconde. En témoignage de ces travaux, le schéma ci-dessous dans la recherche d'Amelia Lambelet et ses collaborateurs explique bien leur résultat : 


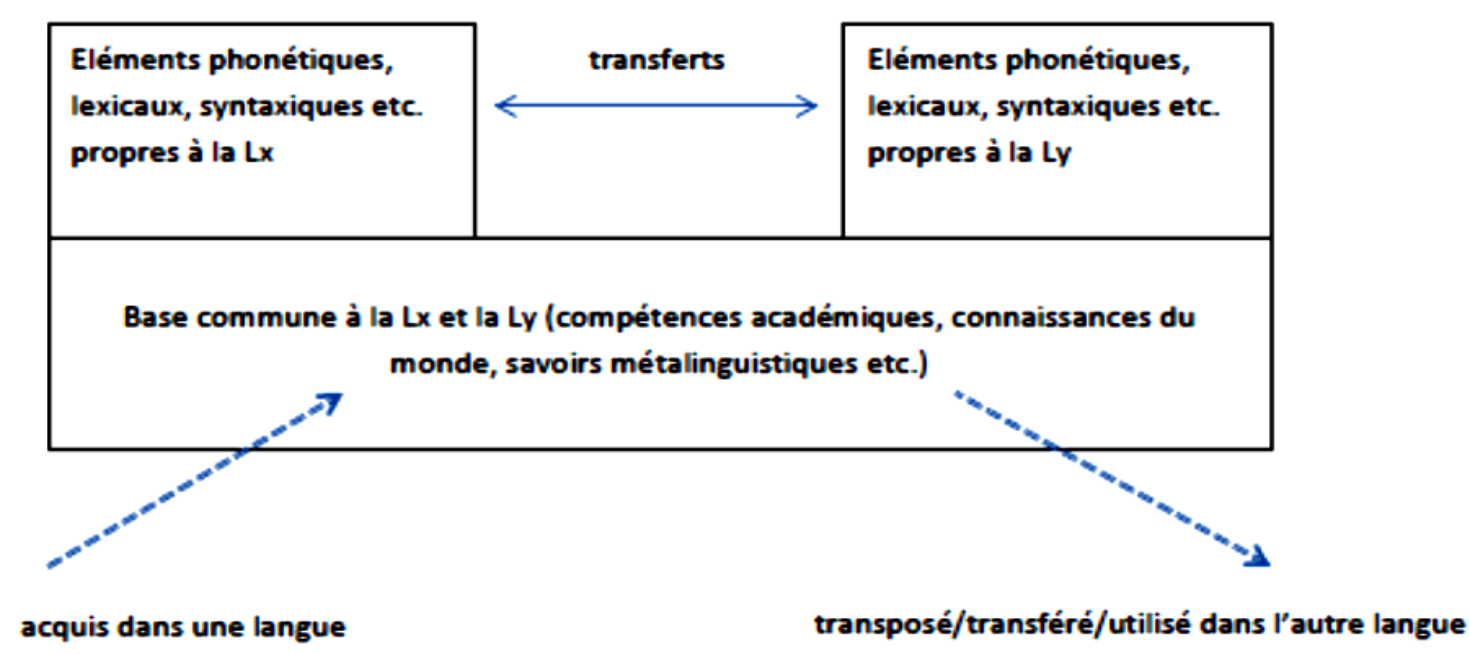

Figure 1. Modélisation graphique des concepts de transferts d'éléments linguistiques et de transferts de compétences formant partie d'une hypothétique base commune aux différentes langues d'un individu plurilingue

Dans notre cas où le persan et l'anglais ont été déjà acquis ou étudiés auparavant, nous nous apercevons que l'apprentissage antérieur de la grammaire persane et la grammaire anglaise peuvent devenir la source de transferts et d'influences sur le français, deuxième langue étrangère. C'est alors que notre problématique s'insère dans le domaine des phénomènes d'interférences et des transferts trilingues. L'analyse contrastive des trois langues vérifie si les sources des connaissances langagières de français prennent racine dans les savoirs préalables en persan ou en anglais.

\subsection{Analyse des erreurs}

Les recherches de Fries soulignent que les matériaux pédagogiques les plus efficaces sont basés sur une description scientifique de la langue à apprendre, comparée avec une description parallèle de la langue maternelle de l'apprenant (9). L'analyse contrastive est la méthode la plus efficace pour comparer des langues. Elle est un outil précieux pour établir les degrés de différence et de ressemblance entre deux ou plusieurs langues, et par conséquent, dans une certaine mesure, le degré de difficulté de l'apprentissage. Selon Henri Besse et Rémy Porquier, les comparaisons linguistiques permettent de prévoir les problèmes d'apprentissage par confrontation des structures phonologiques, synchroniques et lexicosémantiques de la langue « source » et de la langue «cible »; en comparant chaque structure dans les deux systèmes, on peut découvrir tous les problèmes d'apprentissage (200-201). Dans notre cas, s'il y a des ressemblances entre la nouvelle langue (L3) et les langues déjà acquises (L1 et L2), des influences langagières tracent des effets positifs. En revanche, en cas de différences, nous constatons davantage d'obstacles dans l'apprentissage qui pourront être 
les sources des erreurs. Selon Besse et Porquier, ce qui est similaire est facilement transféré, donc facile à apprendre; ce qui est différent donne lieu à un transfert négatif - ou interférence - et donc à des erreurs (206). Développant la théorie de Fries, Robert Lado étudie l'interférence de L1 dans l'apprentissage de L2 et L3 et met en évidence la place du transfert positif ou négatif dans l'apprentissage de langue.

Comme nous avons déjà souligné, notre hypothèse est que les erreurs en français se manifestent sous l'influence de la grammaire persane et/ou celle anglaise. Il nous faut donc une analyse des erreurs du FLE. En effet, l'analyse des erreurs grammaticales aide à proposer des méthodes convenables afin d'améliorer l'apprentissage de FLE. Selon Peter Strevens, l'analyse des erreurs forme une contribution certaine à l'enseignement des langues (85). Notre analyse des erreurs a pour but d'étudier le domaine théorique qui traite le pourquoi et le comment de leurs existences. Elle met en relief d'une part, les différences pour prévenir les risques d'interférences et d'autre part, insiste sur les ressemblances et le degré de proximité afin d'exploiter les potentialités de transfert positif.

Dans nos trois langues considérées, le verbe exprime une action, un état ou un devenir dans la phrase et présente un système complexe de formes (Le Petit Robert Micro, 2013). Il se conjugue, varie en mode, en temps, en voix, en personne et en nombre. Il situe l'action par le temps et la forme dans la durée, soit par rapport au moment où s'exprime le locuteur, soit par rapport à un repère donné dans le contexte, généralement par un autre verbe (Grevisse 1118-1119).

\subsubsection{La place de verbe}

Dans ces trois langues, le verbe possède le même sens ainsi que la même fonction mais sa place dans la phrase est différente. En comparant le couple français/anglais avec le persan, Leila Farkamekh explique que les structures du français et de l'anglais sont signalées avec $S$ $+V+O$, alors qu'en persan, cette structure est signalée autrement $: S+O+V(72)$.

Persan : (Anne + le livre + lit) /Anne ketab ra mikhanad/ (Sujet + Objet + Verbe)

Français : Anne lit le livre. (Sujet + Verbe + Objet)

Anglais : Anne reads the book. (Sujet + Verbe + Objet)

Le sujet, dans ces trois langues, se positionne au début de la phrase. L'objet en persan se place après le sujet et avant le verbe et dans les deux autres, après le verbe. Mais le verbe se met, en français et en anglais, après le sujet. En persan, il est placé à la fin de la phrase, ce qui paraît pour certains peu naturel, car il faut attendre la fin de la phrase pour en comprendre le sens. En persan, quel que soit l'ordre des mots, il faut bien attendre que l'interlocuteur ait fini sa phrase pour en saisir tout le sens. 
Dans cet exemple, l'anglais se manifeste comme langue d'appui du français. La ressemblance structurale entre L2 et L3 pose l'interférence positive qui aide l'apprenant persanophone à généraliser les savoirs de l'anglais au français et à comprendre facilement la syntaxe des phrases françaises. Ainsi, il est capable de distinguer le verbe des autres éléments de la phrase française.

\subsubsection{Accord entre le sujet et le verbe}

Dans la catégorie grammaticale des langues vivantes, certaines règles sont communes, comme celles des pronoms dans le domaine de nombre et le genre (Cormier et al.). Ces ressemblances constituent des bases identiques, même entre des langues éloignées. Pour citer un exemple des ressemblances syntaxiques entre L1 et L3, on peut mentionner la règle grammaticale de l'accord du sujet et du verbe. En persan, en anglais et en français, il existe six formes de pronom personnel, pour six personnes : trois formes de singulier et trois formes de pluriel. Mais les cas ne sont pas aussi nombreux pour le verbe conjugué dans ces trois langues. En français, le verbe varie en personne et en nombre selon, d'une part, que le sujet est, soit à la première personne, soit à la deuxième, soit à la troisième, et d'autre part, que le sujet est au singulier ou au pluriel. En bref, le verbe s'accorde toujours en nombre et en personne avec son sujet (Grevisse 1315). C'est le même cas en persan. À côté des six pronoms personnels, il y a six suffixes, placés après le radical de verbe ; chacun donne un sens au sujet du verbe (Farshidvard 75). En anglais, on a, en général, pour les verbes réguliers deux formes au présent simple : l'une pour les premières, les deuxièmes personnes singulières et plurielles et la troisième personne plurielle, et l'autre pour la troisième personne singulière.

\begin{tabular}{|c|c|c|}
\hline Français & Persan & Anglais \\
\hline Je vais & Man miravam. & I go \\
\hline Tu vas & To miravi. & You go \\
\hline Il /Elle va & Ou miravad. & $\mathrm{He} /$ She goes \\
\hline Nous allons & Ma miravim & We go \\
\hline Vous allez & Shoma miravid. & You go \\
\hline Ils/ Elles vont & Anha miravand. & They go \\
\hline
\end{tabular}

Chez l'apprenant persanophone, l'erreur qui se manifeste, consiste à employer le verbe conjugué à la première personne singulière pour les autres sujets, même si les sujets sont pluriels. Un persanophone produit ainsi les verbes erronés sous l'influence négative de l'anglais : 
Je vais $\rightarrow$ Erreurs : tu vais, illelle vais, nous vais, vous vais, ils/elles vais.

Mais si l'enseignant compare la grammaire des verbes en français et en persan, il peut attirer l'attention sur la similitude de la L1 et de la L3. En effet, en français comme en persan, le verbe s'accorde avec son sujet en nombre. On facilite ainsi le procès d'apprentissage du FLE et évite l'apparition de cette erreur. L'apprentissage antérieur de la grammaire persane devient la source de transferts et des influences positives sur L3.

\subsection{3 « $S$ » ajouté au verbe}

Dans une phrase française où l'apprenant persanophone veut conjuguer le verbe à la troisième personne plurielle, une erreur linguistique est apparue sous l'influence de la grammaire anglaise : en anglais, on utilise le «s » pour construire des noms pluriels de même qu'on ajoute un « $\mathrm{s} »$ au verbe à la troisième personne singulière au présent simple.

Anglais : Paul works. / Paul and David work.

L'apprenant persanophone veut généraliser ses connaissances de l'anglais au français en croyant qu'il est permis d'utiliser le «s $»$ pour changer le verbe au pluriel; il met donc un « $\mathrm{s}$ » au verbe à la troisième personne plurielle.

Français : Paul travaille. / Paul et David travaillent.

Erreur : Paul et David travailles.

Pour éviter cette erreur très répandue, l'enseignant peut profiter de l'interférence du savoir langagier du persan. En persan comme en français, la terminaison ajoutée au radical de verbe désigne la singularité ou la pluralité du verbe tandis qu'en anglais, le verbe ne change pas de terminaisons à la forme singulière ou plurielle sauf dans le présent simple qu'on ajoute un « S » à la troisième personne singulière et ce « S » est la marque du temps du verbe.

Persan : Paul kar mikonad. (Paul travaille) / Paul va David kar mikonnad. (Paul et David travaillent)

Les persanophones font ici référence à leurs connaissances langagières de l'anglais et évitent l'influence de leur langue maternelle, même si cette interférence aide l'apprentissage de FLE.

\subsubsection{Verbe au subjonctif}

En apprenant le subjonctif, une erreur sous l'influence de l'anglais apparait dans la production des phrases françaises où le deuxième verbe doit se conjuguer au subjonctif. Le subjonctif du verbe « être » en persan est /bach/. En anglais, le verbe « to be » garde sa forme infinitive et peut signifier le subjonctif (Darzi et Kwak).

Persan : /Ali mi-xa-d Reza inja baché/.

Anglais : Ali wants Reza to be here.

Mais en français, comme en persan, le deuxième verbe se conjugue au subjonctif. 
Français : Ali veut que Reza soit ici.

Si l'enseignant compare le français et le persan, l'apprenant comprend rapidement la nécessité de conjugaison du deuxième verbe dans une phrase au subjonctif. La ressemblance de la L1 et la L3 exerce donc une influence positive dans ce cas.

\subsection{Synthèse}

Les recherches de Sophie Bailly et ses collaborateurs, réalisées à l'Université de Nancy en France, confirment nos résultats et montrent que la connaissance de l'anglais (première langue étrangère) pourrait constituer un appui dans l'apprentissage/acquisition du français (langue cible et deuxième langue étrangère) par des étudiants de langues maternelles assez éloignées de ces langues européennes. L'apprenant persanophone essaye d'éviter l'exploitation des ressemblances du persan et du français en pensant que l'élément utilisé en français est différent de celui de sa langue maternelle. De plus, il évite automatiquement les règles de sa langue maternelle, même si ces règles se justifient en français. Or, pour Besse et Porquier montrent, ce qui est en « contact » pour l'apprenant à un stade donné d'apprentissage, c'est une grammaire intériorisée de sa langue maternelle et ce qu'il connait, à ce stade, de sa langue étrangère. Des transferts positifs et négatifs peuvent se faire, chez l'apprenant, entre ce qui est déjà acquis de la L1 et de la L2 (203).

Les exemples ci-dessus indiquent les influences de la langue maternelle et de la première langue étrangère sur le français. Dans ces exemples l'enseignant peut jalonner les nouvelles connaissances françaises selon la langue maternelle des apprenants persanophones. Même dans le cas où les langues sont éloignées superficiellement par rapport aux langues voisines, on peut profiter de leurs ressemblances pour enseigner autrement.

\section{En guise de conclusion}

A la suite des contacts linguistiques de l'anglais et du français dans un milieu où la langue maternelle est le persan, on rencontre, comme on peut s'y attendre dans les milieux bilingues ou trilingues, le phénomène d'interférences linguistiques. Nos résultats indiquent que l'interférence manifestée n'est pas seulement liée aux influences de la langue maternelle sur la langue française ; les formes produites par les apprenants dans l'acquisition du français sont influencées aussi par les connaissances préalables de l'anglais. Dans l'apprentissage de FLE, à la suite des interférences linguistiques, les erreurs proviennent des connaissances préalables grammaticales du persan (L1) et/ou de l'anglais (L2). Dans ces conditions, la comparaison de trois langues nous aide : les apprenants avancent plus rapidement dans les premières étapes de l'acquisition de L3 dans le cas où ils ont une perception plus claire des 
lexiques et des règles grammaticales et les comparent à leur langue maternelle tout autant qu'à la première langue étrangère pour généraliser des ressemblances. Les apprenants persanophones du français ont tendance à négliger certaines ressemblances du persan avec le français. Le problème d'interférences linguistiques dans les milieux trilingues fait toujours se manifester des erreurs linguistiques. Notre recherche s'est focalisée sur des erreurs des apprenants persanophones dans l'apprentissage de FLE. Mais, sa portée dépasse largement le cadre de l'Iran, puisque dans de nombreux pays non-anglophones et non-francophones, le français est enseigné comme deuxième langue étrangère après l'anglais. Des études détaillées supplémentaires aideront à mieux comprendre ces interférences et à expliquer des erreurs linguistiques.

\section{Bibliographie}

Bailly, Sophie, et al. «L'anglais langue étrangère : langue d'appui pour l'apprentissage du français langue étrangère ». 〈Hal-00384911v1>, 2009. https://hal.archivesouvertes.fr/hal-00384911v1

Besse, Henri et Rémy Porquier Grammaire et didactique des langues. Paris : Hatier, Coll. Langues et apprentissage des langues (LAL), 1984.

Bild, Eva Rebecca et Merrill Swain. «Minority Language Students in a French Immersion Program : Their French Proficiency ». Journal of Multilingual and Multicultural Development 10.3 (1989). 255-274.

Carrasco-Ortíz, Haydee et Cheryl Frenck-Mestre. « Représentations grammaticales en langue seconde ». TIPA. Travaux interdisciplinaires du laboratoire Parole et langage d'Aixen-Provence 27 (2008). 27-38.

Cenoz, Jasone, Britta Hufeisen et Ulrike Jessner. Cross Linguistic Influence in Third Language Acquisition : Psycholinguistic Perspectives. Clevedon : Multilingual Matters, 2001.

Cormier, Kearsy, Adam Schembri et Bencie Woll. «Pronouns and Pointing in Sign Languages ». Lingua 137 (2013). 230-247.

Darzi, Ali et Saera Kwak. «Syntax and Semantics of Subjunctive Clauses in Persian». Lingua 153 (2015). 1-13.

Dubois, Maud, Alain Kamber et Carine Skupien Dekens. «Tenir compte des langues premières dans l'enseignement / apprentissage des langues vivantes ». Mélanges CRAPEL 35 (2014). 
Farkamekh, Leila. Les influences de l'apprentissage de la première langue étrangère (anglais/L2) sur l'apprentissage de la deuxième langue étrangère (français/L3) chez les apprenants persanophones. Bordeaux : PU Michel de Montaigne - Bordeaux III, 2006.

Frachidvard, Khosro. Grammaire persane contemporaine. Téhéran : Shokhan, 2009.

Fries, Charles Carpenter. Teaching and Learning English as a Foreign Language. Ann Arbor : U of Michigan P, 1945.

Grevisse, Maurice. Le bon usage, grammaire française, refondu par André Goosse. Paris : Duculot, 2006.

Krashen, Stephen et Tracy Terrell. The Natural Approach : Language Acquisition in the Classroom. Londres : Prentice Hall Europe, 1995.

Lado, Robert, Linguistics across Cultures. Ann Arbor : U of Michigan P, 1957.

Lambelet, Amelia, et al. «Acquis dans une langue, transféré dans l'autre ?» Mélanges CRAPEL 35 (2014).

Roux, Pierre Yves. «L'enseignement du français langue étrangère : entre principes et pragmatisme ». Sèvres : CIEP - Centre international d'études pédagogiques, 2014.

Strevens, Peter. «Recherche linguistique et enseignement des langues. Tendances nouvelles en matière de recherche linguistique ». Conseil de la coopération culturelle du conseil de l'Europe 2 Série IV (1964). 85-111. 